\title{
The emergence of specific HDAC inhibitors and their clinical efficacy in the treatment of hematologic malignancies and breast cancer
}

\begin{abstract}
The dynamic balance between histone acetylation and deacetylation is an important epigenetic mechanism controlling gene expression, and is associated with the etiology and progression of many human diseases. Early success of histone deacetylase (HDAC) inhibition in the treatment of hematological cancers paved the way for the development and testing of many different pan/global histone deacetylase inhibitors (HDIs). Dose limiting toxicities in these early trials, coupled with increased understanding of the differential expression patterns of HDACs in different tissues and disease states, led to the emergence of more specific HDIs that target specific HDAC classes and isotypes. Whereas a large number of clinical trials have been undertaken using panHDIs, which have demonstrated varying success in the treatment of hematological and solid malignancies, both as single agents and in combination with other drugs, there have been far fewer undertaken with the emerging repertoire of specific HDIs, which could potentially overcome the limitations seen in the pan inhibitors. In this review we describe the classification and development of HDIs as well the roles of HDACs in cancers, and the rationale behind moving toward more selective inhibition. We then examine the clinical efficacy of both pan- and specific HDI treatment by reviewing a number of clinical trials, focusing on hematological malignancies, where numerous trials have demonstrated single agent and combinatory efficacy, as well as breast cancers, where fewer trials have been undertaken showing limited efficacy, but also where promising pre-clinical findings necessitate further clinical investigation.
\end{abstract}

Keywords: histone deacetylases, histone deacetylase inhibitors, targeted therapy, hematological malignancies, breast cancers
Volume 3 Issue 5 - 2018

\author{
David A Pride, ' Alyssa R Summers ${ }^{1,2,3}$ \\ 'Department of Biology, Sewanee University of the South, \\ Tennessee \\ ${ }^{2}$ Department of Biochemistry, Vanderbilt University School of \\ Medicine, Tennessee \\ ${ }^{3}$ Vanderbilt-Ingram Cancer Center,Vanderbilt University School \\ of Medicine, Tennessee
}

\author{
Correspondence: Alyssa R Summers, Department of Biology, \\ Sewanee:The University of the South, 735 University Ave, 37383 \\ Sewanee Tennessee, Tel (93I)598-I856,
} Email arsummers@sewanee.edu

Received: March 31, 2018| Published: September 26, 2018
Abbreviations: HDACs, histone deacetylase; HDIs, histone deacetylase inhibitors

\section{Introduction}

Epigenetic modification of gene expression through inhibition of histone deacetylases (HDAC) represents a promising potential clinical strategy for a variety of conditions, including cancer, autoimmunity and transplantation, polyglutamine disorders, neurological diseases, and heart disease. ${ }^{1-4}$ Numerous histone deacetylase inhibitors (HDI) have been developed, showing varying degrees of specificity across classes or isotypes of HDACs. Early experience demonstrated the efficacy of less specific, "pan/global" HDIs (e.g. vorinostat, panbinostat, and abexinostat) in the treatment of specific hematological malignancies, but was tempered by significant toxicity exhibited by the inhibitor class. As the preclinical and clinical evaluation of this strategy has progressed, the field has shifted toward a focus on the development and investigation of more specific HDIs ${ }^{5}$ hoping to limit toxicity and unintended non-target effects of HDI. Herein, we seek to review the trend in clinical research toward the development of class and isoformspecific HDIs, highlighting their clinical efficacies and limitations in the treatment of hematologic and breast cancers in order to underscore their potential to allow for the improvement of clinical outcomes with less toxicity and unintended non-target effects.

\section{HDACS and the development of HDIS}

Histone proteins are heavily modified on their N-Terminal tails which play essential role in chromatin architecture and the regulation of gene accessibility and expression. Although many different types of modification take place at these sites, the acetylation is a key regulatory mark that has been most thoroughly investigated. ${ }^{6}$ This acetylation/deacetylation of histone proteins two enzyme families with antagonistic effects: histone acetyl-transferases (HATs) that add acetyl groups to lysine residues, and HDACs that remove these groups. Hyperacetylated histones usually result in transcriptionally active genes, and hypoacetylation generally results in repressed transcription. ${ }^{7}$ Histone proteins are not the only substrates of these enzymes, other proteins have also been revealed to be substrates for reversible acetylation including $\square$-tubulin (HDAC 6 target). ${ }^{6,8,9}$ There are currently 18 human HDACs genes that have been identified and are classified into four distinct families based on their homology to yeast proteins. Class I (homologous to yeast Rpd3), including HDACs $1,2,3$, and 8 , are ubiquitously expressed and are active in complexes with other corepressor proteins such as NCOR and SMRT. ${ }^{10}$ These enzymes are primarily located within the nucleus, with the exception of HDAC3 that has been detected in both the nucleus and cytoplasm. ${ }^{11}$ Class II (homologous to Hdal in yeast) HDACs also can be found in the nucleus as well as the cytoplasm and are divided into two separate subclasses: IIa containing HDACs 4, 5, 7, 9, and IIb containing HDACS 6 , and $10 .{ }^{12}$ Class III (homologous to yeast Sir2) HDACs, termed sirtuins 1-7, are NAD+ dependent and have been found to localize to the nucleus, mitochondria, and cytoplasm. ${ }^{13,14}$ Finally, class IV ("hybrid" sharing similarities to class I, II) consists solely of HDAC $11 . .^{15}$ 
The development of HDIs has resulted in an array of small molecules that target a many key cancer phenotypes including proliferation, apoptosis, differentiation, autophagy, and antiangiogenic effects. ${ }^{16}$ Relatively weak HDAC inhibition was initially observed with sodium butyrate in a clinical setting. This compound served as the prototype of the aliphatic acid group that was later found to include sodium valproate and phenylbutyrate ${ }^{17}$ Following the identification of these compounds a range of much more potent and structurally diverse HDIs have been produced. These compounds are generally classified as hydroxamates, benzamides, tetrapepides/ desmipeptides, and sirtuin inhibitors. ${ }^{18}$

\section{HDACs in cancer}

Cancer results not only from the mutation of genes, but also from changes in epigenetic modifications, such as DNA methylation and histone modification. Unlike genetic mutations, these epigenetic modifications are often reversible. ${ }^{6}$ Because of the ability to potentially change these aberrant epigenetic states, particularly acetylation, that are associated with cancer, HDACs have become viable and promising therapeutic targets. ${ }^{6}$ Atypical expression of HDACs and changes in acetylation levels in human tumors have been demonstrated in numerous correlative studies and overexpression of HDACs 1, 5, and 7 can serve as biomarkers to differentiate tumor from normal tissue. ${ }^{19,20}$ Clearly demonstrated in many studies, abnormal expression and activity of HDACs is correlated to key oncogenic outcomes such as the direct deacetylation of p53 leading to decreased transcriptional activity and HDAC-mediated activation of transcription factors SP1 and C/EBP, which leads to upregulation of oncogenes such as BCL2. 19,21,22 While inappropriate binding of HDACs to promoters has been shown to play a role in tumorigenesis, there is also evidence that fusion proteins created by chromosomal translocations can physically interact with HDACs and lead to deregulated functions that are tumorigenic. $^{6}$

\section{Rationale for selective inhibition}

Despite their relative success in clinical applications, with vorinostat (SAHA, Zolina), and belinostat (PXD101, Beleodaq) that are approved fopr the treatment of hematologic tumors, HDIs that inhibit a broad spectrum of HDACs have all exhibited several significant dose-limiting toxicities (DLT), including fatigue, nausea, vomiting, thrombocytopenia, and neutropenia. ${ }^{5,23}$ In addition, treatment by some HDIs, notably vorinostat, ${ }^{24}$ and panbinostat, ${ }^{25}$ have been associated with serious cardio-toxicity in the treatment of solid cancers. ${ }^{18,26}$ As class II HDACs have been demonstrated to be

Table I Trials of Pan/Global HDIs in Hematoloigical Malignancies abundant in heart tissue, designing inhibitors inactive against class II HDACs could potentially alleviate some of these cardiotoxicities. Due the prevalence of these significant toxicities, increased effort has been directed toward developing HDIs that selectively inhibit certain classes or single isoforms.

In conjunction with the goal of reducing some of the dose limiting toxicities observed with broad-spectrum HDIs, the transition to class- and isotype-specific inhibition could also yield greater therapeutic value. HDACs are frequently over-expressed in many human diseases, predominantly cancers. In particular, class I HDACs are often overexpressed in human tumors, and selective knockdown of HDAC 1 or 2 has been shown to be sufficient to decrease tumor growth. ${ }^{27}$ Accordingly, development of new inhibitors has focused on the inhibition of class I HDACs. While numerous class I specific HDIs have been developed, with many showing preclinical promise, only a few, including the FDA approved romidepsin (FK-228, Istodax), ${ }^{28-30}$ CHR-3966, ${ }^{31}$ entinostat, ${ }^{32}$ mocetinostat ${ }^{33}$ chidamide (CS055/HBI$8000),{ }^{34}$ have entered the clinic. ${ }^{19}$ In addition to these class I specific HDIs, class I and II specific HDIs, including AR- $42^{35}$ and the hydroxamide quisinostat (JNJ26481585) ${ }^{36}$ have been developed and put to clinical trial. ${ }^{19}$

\section{Treatment of hematologic malignancies with HDI}

Unlike many types of solid cancers, hematologic malignancies have proved particularly sensitive to HDI treatment (Table 1). The first disease to have an FDA designated HDI as a treatment option, following failed attempts of other systemic treatments, was cutaneous T-cell lymphoma (CTCL). CTCL consists of extranodal non-Hodgkin's lymphomas (NHLs) made up of malignant, clonal T-cells presenting in the skin. Numerous phase I and II clinical trials have been undertaken for CTCL using the panHDIs vorinostat, ${ }^{37,38}$ panbinostat, ${ }^{39}$ and belinostat, ${ }^{40,41}$ both as single agents and in combination with other chemotherapeutic compounds. Notably, panbinostat has been investigated in patients after treatment with the retinoid bexarotene, ${ }^{42}$ and belinostat has been investigated in combination with many agents including bortezomib, 5-fluorouracil, 5-azacytidine, carboplatin, paclitaxel, and isoretinoin. ${ }^{43}$ These drugs were generally well tolerated in CTCL patients as well as in peripheral T-cell lymphoma (PTCL), ${ }^{44,45}$ Hodgkin's lymphoma, ${ }^{46-48}$ multiple myeloma, ${ }^{49,50}$ diffuse B-cell lymphoma, ${ }^{51}$ myelodysplastic syndrome, ${ }^{52}$ and acute myeloid leukemia, ${ }^{53}$ often achieving complete remission (CR), partial remission (PR), and sustained disease (SD), while exhibiting manageable dose limiting toxicities such as fatigue, nausea, anemia, and vomiting. ${ }^{43}$

\begin{tabular}{|c|c|c|c|c|c|c|}
\hline Agent & Combinations & Disease & Phase & Number & Response & Citation \\
\hline Vorinostat & Single agent & CTCL & II & 33 & PR 8 & 37 \\
\hline Vorinostat & Single agent & CTCL & $\mathrm{llb}$ & 74 & CR I, PR 2I & 38 \\
\hline Vorinostat & Single agent & Hodgkin's Lymphoma & ॥ & 25 & PR I, SD 7 & 47 \\
\hline Vorinostat & Single agent & $\begin{array}{l}\text { Advanced Leukemias, and } \\
\text { Myelodyspastic syndrome }\end{array}$ & I & 29 & CR 3 & 52 \\
\hline Vorinostat & $\begin{array}{l}\text { Carfilzomib, } \\
\text { lenalidomide, } \\
\text { dexamthasone }\end{array}$ & MM & I & 17 & $\begin{array}{l}\text { PR 9, } 5 \text { SD, } \\
\text { PD } 2\end{array}$ & 50 \\
\hline Panbinostat & Single agent & Hodgkin's Lymphoma & ॥ & 129 & PR 30, CR 5 & 48 \\
\hline
\end{tabular}


Table Continued

\begin{tabular}{|c|c|c|c|c|c|c|}
\hline Agent & Combinations & Disease & Phase & Number & Response & Citation \\
\hline Panbinostat & Bexarotene & CTCL & II & 118 & CR/PR 24 & 42 \\
\hline Belinostat & Single agent & PTCL & II & 31 & $\begin{array}{l}\text { CR I3, PR } \\
18\end{array}$ & 45 \\
\hline Belinostat & Single agent & CTCL/PTCL & II & $\begin{array}{l}\text { CR, PR, } \\
\text { SD, PD }\end{array}$ & $\begin{array}{l}\text { CR, PR, SD, } \\
\text { PD }\end{array}$ & 41 \\
\hline Abexinostat & Single agent & Relapsed lymphoma & $\mathrm{I} / \mathrm{II}$ & 21 & CR 2, PR I4 & 85 \\
\hline
\end{tabular}

Abbreviations: SD, sustained disease; CR, complete remission; PR, partial remission; DR, durable response;WT, well-tolerated; NR, no response; CTCL, cutaneous T-cell lymphoma; AML, acute myeloid leukemia; PTCL, peripheral T-cell lymphoma; MM, multiple myeloma.

Whereas hundreds of clinical trials have been undertaken with panHDIs in the treatment of hematologic malignancies, fewer have been undertaken with class and isoform specific HDIs (Table 2). In order to investigate the efficacy of these specific HDIs in treating hematologic malignancies, we review the results of a selection of clinical trials.

Table 2 Trials of Specific HDls in Hematological Malignancies

\begin{tabular}{|c|c|c|c|c|c|c|}
\hline Agent & Combinations & Disease & Phase & Number & Response & Citation \\
\hline Romidepsin & Single agent & CTCL & II & 71 & CR I, PR 20 & 57 \\
\hline Romidepsin & Single agent & CLL,AML & I & 20 & $N R$ & 53 \\
\hline Romidepsin & Single agent & CTCL & II & 96 & CR 5, PR 2I & 56 \\
\hline Romidepsin & Single agent & PTCL & II & 45 & CR 8, PR 9 & 55 \\
\hline Entinostat & Single agent & $\begin{array}{l}\text { Lymphoid malignancies and refractory solid } \\
\text { tumors }\end{array}$ & I & 22 & WT & 32 \\
\hline Chidamide & Single agent & Advanced solid tumors, lymphomas & II & 31 & PR 5 & 34 \\
\hline AR-42 & SIngle agent & Relapsed MM, lymphoma & I & 17 & WT & 35 \\
\hline Mocentinostat & Single agent & lymphocytic leukemia & II & 21 & NR & 33 \\
\hline
\end{tabular}

Abbreviations: SD, sustained disease; CR, complete remission; PR, partial remission; DR, durable response;WT, well-tolerated; NR, no response; CTCL, cutaneous T-cell lymphoma;AML, acute myeloid leukemia; PTCL, peripheral T-cell lymphoma; MM, multiple myeloma.

Romidepsin is a bicyclic peptide that, although originally developed for its ability to reverse the ras-transformed phenotype to normal, was found to potently inhibit class I HDACs in $1998 .{ }^{29}$ Phase I and II clinical trials initiated by the National Cancer Institute achieved therapeutic responses in patients with CTCL and PTCL. ${ }^{44,53,54}$ Phase II studies into the efficacy of romidepsin were conducted using a dosage of $14 \mathrm{mg} / \mathrm{m}^{2}$ infused over four hours on days 1,8 , and 15 of a 8 -day cycle, and resulted in a number of PRs. ${ }^{29,44}$ Further phase II studies confirmed this efficacy achieving both CR and PR in CTCL and PTCL patients. ${ }^{55,56}$ These successes were tarnished by the sudden cardiac death of two patients in a trial conducted by Piekarz et al. ${ }^{44}$ which indicated cardio-toxicity as observed in panHDIs. ${ }^{29,57}$ This finding, however, led to a systematic study into the potential cardiac effects of romidepsin treatment, which confirmed the drug's safety. ${ }^{30}$

Entinostat (MS-275), selectively inhibits class I HDACs and exhibited promising pre-clinical results showing not only potent antiproliferative functions via the induction of(CIP1/WAF1), but also increased expression of differentiation markers (CD11b) and induction of apoptosis via increases in reactive oxygen species, mitochondrial damage, and caspase activation..$^{19,32,58}$ A phase I clinical trial also established that unlike other HDI treatments, treatment with Entinostat resulted in no detectable cardiac events..$^{32}$ Investigation into the combination treatment of myeloid malignancies with Entinostat and 5-Azacytidine in which, participants were treated for ten consecutive days with various doses of 5-Azacytidine and received Entinostat orally on intermittent days on a 28 or 29 day cycle., ${ }^{6,59}$ Within the group of 30 patients who received at least 4 therapy cycles, three had a complete remission, four had a partial remission, and seven showed hematologic improvements. ${ }^{59}$ Alone or in combination with standard therapies HDIs are showing promise.

Chidamide (CS055/HBI-8000) represents a benzamide class HDI that is designed to inhibit the catalytic site of class I HDACs, while exhibiting improved metabolic stability as compared to other hydroxamic acid and benzamide type inhibitors. ${ }^{34,60,61}$ Studies into enzymatic inhibition by chidamide have shown that it effectively inhibits class I HDACS and class II HDAC $10 . .^{34,61}$ In addition, preclinical studies have shown broad-spectrum in vitro and in vivo anti-tumor activity as well as oral bioavailabilty with chidamide treatment. ${ }^{34,61}$ A phase I clinical trial was performed on a cohort of 31 patients with advanced solid tumors or lymphomas where doses between 5 to $50 \mathrm{mg}$ chidamide were administered several times per week for four consecutive weeks every six weeks. Overall, the treatments were well tolerated within the trial period. Chidamide was also shown to display favorable pharmokinetic and pharmodynamic qualities that had a relatively long half-life while demonstrating significant preliminary anti-tumor activity. ${ }^{34}$ Finally, consistent with previous clinical studies that demonstrated HDI's significant single-agent anti-tumor activity in lymphomas, chidamide treatment produced four partial responses out of five patients enrolled with T-cell lymphoma, while also producing partial responses in a patient with adenoid cystic carcinoma of the submandibular gland. ${ }^{34,38,56}$

Mocetinostat is an isotype-specific aminophenylbenzamide that inhibits HDAC classes I and IV with very little class II inhibition that has been involved in different phase I and phase II studies for hematologic malignancies. ${ }^{62}$ The results of a phase II trial in patients with advanced chronic lymphocytic leukemia (CLL), where patients received 
mocetinostat with an escalating dose or the addition of rituximab was permitted after two or more cycles without response, demonstrated limited anti-cancer activity as no responses were observed. ${ }^{33}$ The limitations of mocetinostat use in CLL were additionally apparent with the observation of several toxicities including infections, thrombocytopenia, anemia, diarrhea, and fatigue. ${ }^{6,33}$ These findings demonstrate the need for further investigations into combinatory treatments with mocetinostat. ${ }^{6}$ However, a later phase II trial in the treatment of classical Hodgkin's lymphoma showed mocetinostat as a promising single-agent with controllable toxicity in patients with relapsed classical Hodgkin's lymphoma. ${ }^{63}$

\section{HDIS in breast cancer}

No longer viewed as a single disease, breast cancer consists of a diverse group of tumors and is the most common cancer diagnosed in women worldwide. ${ }^{64}$ The heterogeneity of breast tumors has long been observed in histological and clinical outcomes, however, recent advances in molecular biology have allowed for more refined classifications that link molecular characteristics with disease mechanisms to clinical outcomes. ${ }^{65,66}$ Comprehensive gene expression profiling has allowed for the classification of breast tumors into at several major subtypes each with their own risk factors, treatment responses, risk of disease progression, and organ sites of metastases. ${ }^{65}$ Luminal tumors are positive for estrogen (ER) and progesterone receptors (PR) and respond well to hormonal interventions. Human epidermal growth receptor $2+(\mathrm{HER} 2+)$ tumors are characterized by overexpression of the ERBB2 oncogene, and have been effectively

Table 3 Trials of Pan/Global HDIs in Breast Cancer controlled with a diverse array of anti-HER2 therapies. Finally, basal like tumors in general lack hormone receptors and HER2 and are therefore referred to as triple-negative breast cancer (TNBC). ${ }^{67,68}$ This group, which is one of the highest priority areas of research in breast cancer, responding approximately $20 \%$ of the time to standard chemotherapy, can further be divided into additional subclasses, each with its own molecular features and sensitivity to treatment. ${ }^{65}$

Corresponding with other solid cancers, but contrasting hematologic malignancies, far fewer clinical trials have been undertaken using HDIs in the treatment of breast cancer. Phase I and II clinical trials thus far have focused on the panHDIs vorinostat, ${ }^{69-71}$ and panbinostat ${ }^{72}$ in metastatic breast cancer (Table 3). Further contrasting the use of HDIs in hematologic malignancies, nearly all HDI regimens in breast cancer treatments are administered in combination with other chemotherapeutic drugs. Vorinostat has been combined with paclitaxel and bevacizumab, ${ }^{70}$ and tamoxifen ${ }^{73}$ in phase I and II trials, where treatment regimens have been well tolerated with response rates of approximately 40 percent. Panbinostat has been combined with letrozole in a phase I trial in postmenopausal metastatic breast cancer, with a moderate response showing 2 and 5 of 12 participants experiencing PR and SD respectively. ${ }^{72}$ Vorinostat has also been investigated as a single-agent treatment in breast cancer, however response rates were poor. ${ }^{69}$ These trials demonstrated that, although HDI has not been seen to be particularly effective in the treatment of breast cancer as single-agents, the promise that combinatory treatments exhibit necessitates further investigation.

\begin{tabular}{lllllll}
\hline Agent & Combinations & Disease & Phase & Number & Response & Citation \\
\hline Vorinostat & Single agent & Metastatic breast cancer & II & I4 & SD I & 69 \\
Vorinostat & $\begin{array}{l}\text { Paclitaxel, } \\
\text { bevacizumab }\end{array}$ & Metastatic breast cancer & I/I & 44 & $\begin{array}{l}\text { CR 2, PR 24, } \\
\text { SD 16 }\end{array}$ & 70 \\
Vorinostat & Tamoxifen & $\begin{array}{l}\text { Hormone-therapy resistant breast } \\
\text { cancer }\end{array}$ & II & 43 & DR 8, SD 9 & 73 \\
Panbinostat & Letrozole & $\begin{array}{l}\text { Post-menopausal metastatic breast } \\
\text { cancer }\end{array}$ & I & I2 & PR 2, SD 5 & 72 \\
\hline
\end{tabular}

Abbreviations: SD, sustained disease; CR, complete remission; PR, partial remission; DR, durable response.

Although the efficacy of HDI in breast cancer clinical trials has not been demonstrated quite as extensively as in hematological malignancies, numerous preclinical studies have implicated HDACs as potential therapeutic targets. Treatment of TNBC cell lines MDA-MB-157, MDA-MB-231, MDA-MB-468, and BT-549 with panbinostat resulted in decreased cell proliferation and survival, apoptosis induction (excluding MDA-MB-468), and induced changes in cell morphology consistent with reversal from the mesenchymal phenotype. ${ }^{74}$ Additionally, panbinostat treatment, in combination with salinomycin, of TNBC cells and an in vivo xenograft mouse model synergistically inhibited cell proliferation through the induction of apoptosis, cell cycle arrest, and epithelial mesenchymal transition (EMT) regulation. ${ }^{75}$ These findings implicate both single-agent and combinatory efficacy for HDI treatment in TNBC, although these results have been tempered by limited results in a phase II trial of entinostat. $^{76}$ Further evidencing the promise of HDI treatment in combination with other drugs, the inhibitor LAQ824 was demonstrated to down-regulate HER2 and sensitize breast cancer cells to trastuzumab, taxotere, gemcitabine, and epothilone B. ${ }^{77}$

Due to observed overexpression of class I HDACs 1, 2, and 3 in varying breast tumor types much of the research on HDI in breast cancer has focused on the class I HDACs and their inhibitors. ${ }^{78}$ HDACs 2 and 3 expression was demonstrated to be higher in less differentiated tumors and was correlated with the negative hormone receptor expression phenotype associated with more aggressive tumor types, ${ }^{78}$ Concordantly, HDAC 2 overexpression was significantly correlated with HER2 expression and nodal metastasis. In contrast, HDAC 1 was highly expressed in hormone receptor positive tumors, which generally exhibit less aggressive features. ${ }^{78}$ This finding, however, conflicts with earlier experimental evidence showing negative regulation of the estrogen receptor alpha (ER) by HDAC $1 .^{79}$ In addition to HDACs 1,2 , and 3 the class I HDAC 8 has been implicated in the progression of breast cancer. Upregulated HDAC 8 expression was demonstrated in paired breast cancer tissue from both a TCGA data set and a cohort of Taiwanese patients, and was associated with poor prognosis in early-stage breast cancer, latestage disease, and tumor progression..$^{80}$ Knockdown by si-HDAC8 and inhibition with the novel HDAC 8 inhibitor PCI-34051 markedly reduced (by $89.0 \%$ ) cell migration, as evidenced in both Transwell and wound-healing assays, in the highly invasive breast cancer cell 
line MDA-MB-231.

In addition to the class I HDACs, class II HDACs 5 and 9 have been implicated as potential therapeutic targets in breast cancer. ${ }^{81,82}$ HDAC 5 overe xpression has been demonstrated in human breast cancer cell lines, where it functionally interacts with lysine-specific demethylase 1 (LSD1) to promote cancer progression. Depletion of HDAC 5 in MDA-MB-231 cells resulted in decreased cellular LSD1 levels, hindered cellular proliferation, induced G1 cell-cycle arrest, and attenuated cell migration and colony formation. ${ }^{81}$ Similarly, HDAC 9 overexpression was shown in highly aggressive basal breast cancer cell lines, as well as basal tumors, and was associated with higher rates of gene transcription and epigenetic marking of the HDAC 9 promoter. ${ }^{82}$ Ectopic expression of HDAC 9 in luminal breast cancer cells resulted in increased cell proliferation and decreased apoptosis. These effects, associated with deregulated expression of HDI controlled genes such as CDKN1A, BAX, and TNFRSF10A, were reversed in basal breast cancer cells after HDAC 9 knockdown. ${ }^{82}$ These findings implicate the involvement of HDACs 5 and 9 in the regulation of mammary carcinogenesis and HDI response, and could potentially serve as targets in personalized HDI treatment.

\section{Conclusion}

Histone deacetylase inhibition represents a promising epigenetic strategy for the treatment of a number of diseases, in particular in hematologic malignancies and other cancers. Following the early successes of panHDIs in the treatment of hematologic malignancies, and the subsequent discovery of dose-limiting toxicities and unintended non-target effects of the same, there has been a trend toward the development of class- and isoform-specific HDIs that can be individualized toward specific diseases, improving patient outcomes while limiting the drugs' toxic effects. ${ }^{5}$ While these novel HDIs have been demonstrated to have higher potencies in preclinical investigation, and have produced positive results in early clinical trials, further investigation is needed in order to determine if selective HDAC inhibition will remain effective at treating disease with minimal adverse effects. ${ }^{43}$ The clinical success of single agent and combinatory HDI treatments of hematologic malignancies stands in contrast to the limitations of HDI treatment in solid cancers, particularly breast cancer. However, strong preclinical evidence has been elucidated implicating the potential for selective HDI success in breast cancer. ${ }^{74-78,80-84}$ The contrast between the success of panHDI in the treatment of hematologic malignancies, where selective HDI treatments have only showed modestly improved outcomes, with the newly emerging implications for success in selective HDI treatment in breast cancer, demonstrates the need for further clinical investigation into class/isoform specific HDAC inhibition.

\section{Acknowledgments}

None.

\section{Conflict of interests}

The authors have declared that no conflict of interest exists.

\section{References}

1. Hancock WW, Akimova T, Beier UH, et al. HDAC inhibitor therapy in autoimmunity and transplantation. Ann Rheum Dis. 2012;71 (Suppl 2):46-54.

2. Thomas EA. Involvement of HDAC1 and HDAC3 in the Pathology of Polyglutamine Disorders: Therapeutic Implications for Selective HDAC1/HDAC3 Inhibitors. Pharmaceuticals (Basel). 2014;7(6):634 641 .

3. Falkenberg KJ, Johnstone RW. Histone deacetylases and their inhibitors in cancer, neurological diseases and immune disorders. Nat Rev Drug Discov. 2014;13(9):673-691.

4. McKinsey TA. Isoform-selective HDAC inhibitors: closing in on translational medicine for the heart. $J$ Mol Cell Cardiol. 2011;51(4):491-496.

5. Li X, Xu W. HDAC1/3 dual selective inhibitors - new therapeutic agents for the potential treatment of cancer. Drug Discov Ther. 2014;8(5):225-228.

6. Wagner JM, Hackanson B, Lubbert M, et al. Histone deacetylase (HDAC) inhibitors in recent clinical trials for cancer therapy. Clin Epigenetics.2010;1(3-4):117-136

7. Redner RL, Wang J, Liu JM. Chromatin remodeling and leukemia: new therapeutic paradigms. Blood. 1999;94(2):417-428.

8. Buchwald M, Kramer OH, Heinzel T. HDACi - Targets beyond chromatin. Cancer Lett. 2009;280(2):160-167.

9. Glozak MA, Sengupta N, Zhang X, et al. Acetylation and deacetylation of non-histone proteins. Gene. 2005;363:15-23.

10. Roth SY, Denu JM, Allis CD. Histonea cetyltransferases. Annu Rev Biochem. 2001;70:81-120.

11. De Ruijter AJM, Van Gennip AH, Caron HN, et al. Histone deacetylases (HDACs): characterization of the classical HDAC family. Biochem $J$. 2003;370(Pt 3):737-749.

12. Verdin E, Dequiedt F, Kasler HG. Class II histone deacetylases: versatile regulators. Trends Genet. 2003;19(5):286-293.

13. Taylor DM, Maxwell MM, Luthi-Carter R, et al. Biological and Potential Therapeutic Roles of Sirtuin Deacetylases. Cell Mol Life Sci. 2008;65(24):4000-4018.

14. Michishita E, Park JY, Burneskis JM, et al. Evolutionarily conserved and nonconserved cellular localizations and functions of human SIRT proteins. Mol Biol Cell. 2005;16(10):4623-4635.

15. Gao L, Cueto MA, Asselbergs F, et al. Cloning and functional characterization of HDAC11, a novel member of the human histone deacetylase family. J Biol Chem.2002;277(28):25748-25755.

16. Marks PA, Richon VM, Breslow R, et al. Histone deacetylase inhibitors as new cancer drugs. Curr Opin Oncol. 2001;13(6):477-483.

17. Prince HM, Bishton MJ, Harrison SJ. Clinical studies of histone deacetylase inhibitors. Clin Cancer Res. 2009;15(12):3958-3969.

18. Benedetti R, Conte M, Altucci L. Targeting Histone Deacetylases in Diseases: Where Are We? Antioxid Redox Signal. 2015;23(1):99-126.

19. West AC, Johnstone RW. New and emerging HDAC inhibitors for cancer treatment. J Clin Invest. 2014;124(1):30-39.

20. Özdağ H, Teschendorff AE, Ahmed AA, et al. Differential expression of selected histone modifier genes in human solid cancers. BMC Genomics. 2006;25(7):90.

21. Luo J, Su F, Chen D, et al. Deacetylation of p53 modulates its effect on cell growth and apoptosis. Nature. 2000;408(6810):377-381.

22. Duan H, Heckman CA, Boxer LM. Histone deacetylase inhibitors down-regulate bcl-2 expression and induce apoptosis in $\mathrm{t}(14 ; 18)$ lymphomas. Mol Cell Biol. 2005;25(5):1608-1619.

23. Tan J, Cang S, Ma Y, et al. Novel histone deacetylase inhibitors in 
clinical trials as anti-cancer agents. J Hematol Oncol. 2010. p. 5.

24. Zhong HM, Ding QH, Chen WP, et al. Vorinostat, a HDAC inhibitor, showed anti-osteoarthritic activities through inhibition of iNOS and MMP expression, p38 and ERK phosphorylation and blocking NF- $\kappa \mathrm{B}$ nuclear translocation. Int Immunopharmacol. 2013;17(2):329-335.

25. De Marinis F, Atmaca A, Tiseo M, et al. A Phase II Study of the Histone Deacetylase Inhibitor Panobinostat (LBH589) in Pretreated Patients with Small-Cell Lung Cancer. J Thorac Oncol. 2013;8(8):1091-1094.

26. Shultz MD, Cao X, Chen $\mathrm{CH}$, et al. Optimization of the in Vitro Cardiac Safety of Hydroxamate-Based Histone Deacetylase Inhibitors. J Med Chem. 2011;54(13):4752-4772.

27. Stubbs MC, Kim W, Bariteau M, et al. Selective Inhibition of HDAC1 and HDAC2 as a Potential Therapeutic Option for B-ALL. Clin Cancer Res. 2015;21(10):2348-2358.

28. Amiri Kordestani L, Luchenko V, Peer CJ, et al. Phase I trial of a new schedule of romidepsin in patients with advanced cancers. Clin Cancer Res. 2013;19(16):4499-4507.

29. McGraw AL. Romidepsin for the treatment of T-cell lymphomas. Am $J$ Health Syst Pharm. 2013;70(13):1115-1122.

30. Noonan AM, Eisch RA, Liewehr DJ, et al. Electrocardiographic studies of romidepsin demonstrate its safety and identify a potential role for K(ATP) channel. Clin Cancer Res. 2013;19(11):3095-3104.

31. Banerji U, Van Doorn L, Papadatos Pastos D, et al. A phase pharmacokinetic and pharmacodynamic study of CHR-3996, an oral class I selective histone deacetylase inhibitor in refractory solid tumors. Clin Cancer Res. 2012;18(9):2687-2694.

32. Kummar S, Gutierrez M, Gardner ER, et al. Phase I Trial of MS-275, a Histone Deacetylase Inhibitor, Administered Weekly in Refractory Solid Tumors and Lymphoid Malignancies. Clin Cancer Res. 2007;13(18 Pt 1):5411-5417.

33. Blum KA, Advani A, Fernandez L, et al. Phase II study of the histone deacetylase inhibitor MGCD0103 in patients with previously treated chronic lymphocytic leukaemia. Br J Haematol. 2009;147(4):507514.

34. Dong $M$, Ning ZQ, Xing PY, et al. Phase I study of chidamide (CS055/HBI-8000), a new histone deacetylase inhibitor, in patients with advanced solid tumors and lymphomas. Cancer Chemother Pharmacol. 2012;69(6):1413-1422.

35. Hofmeister CC, Liu Z, Bowers MA, et al. Phase I Study of AR-42 in Relapsed Multiple Myeloma and Lymphoma. Blood. 2012;120:2955.

36. Carol H, Gorlick R, Kolb EA, et al. Initial testing (stage 1) of the histone deacetylase inhibitor, quisinostat (JNJ-26481585), by the Pediatric Preclinical Testing Program. Pediatr Blood Cancer. 2014;61(2):245-252.

37. Duvic M, Talpur R, Ni X, et al. Phase 2 trial of oral vorinostat (suberoylanilide hydroxamic acid, SAHA) for refractory cutaneous T-cell lymphoma (CTCL). Blood. 2007;109(1):31-39.

38. Olsen EA, Kim YH, Kuzel TM, et al. Phase IIb multicenter trial of vorinostat in patients with persistent, progressive, or treatment refractory cutaneous T-cell lymphoma. J Clin Oncol. 2007;25(21):3109-3115.

39. Miles Prince H, Bishton M, Harrison S. The potential of histone deacetylase inhibitors for the treatment of multiple myeloma. Leuk Lymphoma. 2008;49(3):385-387.

40. Howman RA, Prince HM. New drug therapies in peripheral T-cell lymphoma. Expert Rev Anticancer Ther. 2011;11(3):457-472.

41. Foss F, Advani R, Duvic M, et al. A Phase II trial of Belinostat
(PXD101) in patients with relapsed or refractory peripheral or cutaneous T-cell lymphoma. Br J Haematol. 2015;168(6):811-819.

42. Duvic M, Dummer R, Becker JC, et al. Panobinostat activity in both bexarotene-exposed and -naïve patients with refractory cutaneous T-cell lymphoma: Results of a phase II trial. European Journal of Cancer.2013;49(2):386-394

43. Stimson L, Wood V, Khan O, et al. HDAC inhibitor-based therapies and haematological malignancy. Ann Oncol. 2009;20(8):1293-1302.

44. Piekarz RL, Robey R, Sandor V, et al. Inhibitor of histone deacetylation, depsipeptide (FR901228), in the treatment of peripheral and cutaneous T-cell lymphoma: a case report. Blood. 2001;98(9):2865-2868.

45. OConnor OA, Horwitz S, Masszi T, et al. Belinostat in Patients With Relapsed or Refractory Peripheral T-Cell Lymphoma: Results of the Pivotal Phase II BELIEF (CLN-19) Study. J Clin Oncol. 2015;33(23):2492-2499.

46. Oki Y, Copeland A, Younes A. Clinical development of panobinostat in classical Hodgkin's lymphoma. Expert Rev Hematol. 2011;4(3):245252 .

47. Kirschbaum MH, Goldman BH, Zain JM, et al. A phase 2 study of vorinostat for treatment of relapsed or refractory Hodgkin lymphoma: Southwest Oncology Group Study S0517. Leuk Lymphoma. 2012;53(2):259-262.

48. Younes A, Sureda A, Ben Yehuda D, et al. Panobinostat in patients with relapsed/refractory Hodgkin's lymphoma after autologous stem-cell transplantation: results of a phase II study. J Clin Oncol. 2012;30(18):2197-2203.

49. Kaufman JL, Fabre C, Lonial S, et al. Histone deacetylase inhibitors in multiple myeloma: rationale and evidence for their use in combination therapy. Clin Lymphoma Myeloma Leuk. 2013;13(4):370-376.

50. Vesole DH, Bilotti E, Richter JR, et al. Phase I study of carfilzomib, lenalidomide, vorinostat, and dexamethasone in patients with relapsed and/or refractory multiplemyeloma.BrJHaematol.2015;171(1):52-59.

51. De Paepe P, De Wolf Peeters C. Diffuse large B-cell lymphoma: a heterogeneous group of non-Hodgkin lymphomas comprising several distinct clinicopathological entities. Leukemia. 2007;21(1):37-43.

52. Garcia Manero G, Yang H, Bueso Ramos C, et al. Phase 1 study of the histone deacetylase inhibitor vorinostat (suberoylanilide hydroxamic acid [SAHA]) in patients with advanced leukemias and myelodysplastic syndromes. Blood. 2008;111(3):1060-1066.

53. Byrd JC, Marcucci G,Parthun MR, etal.Aphase 1 and pharmacodynamic study of depsipeptide (FK228) in chronic lymphocytic leukemia and acute myeloid leukemia. Blood. 2005;105(3):959-967.

54. Marshall JL, Rizvi N, Kauh J, et al. A phase I trial of depsipeptide (FR901228) in patients with advanced cancer. J Exp Ther Oncol. 2002;2(6):325-332.

55. Piekarz RL, Frye R, Prince HM, et al. Phase 2 trial of romidepsin in patients with peripheral T-cell lymphoma. Blood. 2011;117(22):58275834 .

56. Whittaker SJ, Demierre MF, Kim EJ, et al. Final results from a multicenter, international, pivotal study of romidepsin in refractory cutaneous T-cell lymphoma. J Clin Oncol. 2010;28(29):4485-4491.

57. Piekarz RL, Frye R, Turner M, et al. Phase II multi-institutional trial of the histone deacetylase inhibitor romidepsin as monotherapy for patients with cutaneous T-cell lymphoma. J Clin Oncol.2009;27(32):5410-5417.

58. Rosato RR, Almenara JA, Grant S. The histone deacetylase inhibitor MS-275 promotes differentiation or apoptosis in human leukemia cells through a process regulated by generation of reactive oxygen species 
and induction of p21CIP1/WAF1 1. Cancer Res. 2003;63(13):36373645 .

59. Fandy TE, Herman JG, Kerns P, et al. Early epigenetic changes and DNA damage do not predict clinical response in an overlapping schedule of 5-azacytidine and entinostat in patients with myeloid malignancies. Blood. 2009;114(13):2764-2773.

60. Yin ZH, Wu ZW, Lan YK, et al. Synthesis of chidamide, a new histone deacetylase (HDAC) inhibitor. Chin J New. 2004.

61. Ning ZQ, Li ZB, Newman MJ, et al. Chidamide (CS055/HBI-8000): a new histone deacetylase inhibitor of the benzamide class with antitumor activity and the ability to enhance immune cell-mediated tumor cell cytotoxicity. Cancer Chemother Pharmacol. 2012;69(4):901-909.

62. Boumber Y, Younes A, Garcia Manero G. Mocetinostat (MGCD0103): a review of an isotype-specific histone deacetylase inhibitor. Expert Opin Investig Drugs. 2011;20(6):823-829.

63. Younes A, Oki Y, Bociek RG, et al. Mocetinostat for relapsed classical Hodgkin's lymphoma: an open-label, single-arm, phase 2 trial. Lancet Oncol. 2011;12(13):1222-1228.

64. Hortobagyi GN. Trastuzumab in the Treatment of Breast Cancer. $N$ Engl J Med 2005;353:1734-1736.

65. Polyak K. Heterogeneity in breast cancer. $J$ Clin Invest 2011;121(10):3786-3788.

66. Perez EA. Breast cancer management: opportunities and barriers to an individualized approach. Oncologist. 2011;16(Suppl 1):20-22.

67. Sørlie T, Perou CM, Tibshirani R, et al. Gene expression patterns of breast carcinomas distinguish tumor subclasses with clinical implications. Proc Natl Acad Sci USA. 2001;98(19):10869-10874.

68. Perou CM, Sørlie T, Eisen MB, et al. Molecular portraits of human breast tumours. Nature.2000;406(6797):747-752.

69. Luu TH, Morgan RJ, Leong L, et al. A phase II trial of vorinostat (suberoylanilide hydroxamic acid) in metastatic breast cancer: a California Cancer Consortium study. Clin Cancer Res. 2008;14(21):7138-7142.

70. Ramaswamy B, Fiskus W, Cohen B, et al. Phase I-II study of vorinostat plus paclitaxel and bevacizumab in metastatic breast cancer: evidence for vorinostat-induced tubulin acetylation and Hsp90 inhibition in vivo. Breast Cancer Res Treat. 2012;132(3):1063-1072.

71. Munster PN, Thurn KT, Thomas S, et al. A phase II study of the histone deacetylase inhibitor vorinostat combined with tamoxifen for the treatment of patients with hormone therapy-resistant breast cancer Br J Cancer.2011;104(12):1828-1835.

72. Tan WW, Allred JB, Moreno Aspitia A, et al. Phase I Study of
Panobinostat (LBH589) and Letrozole in Postmenopausal Metastatic Breast Cancer Patients. Clin Breast Cancer. 2016;16(2):82-86.

73. Tate CR, Rhodes LV, Segar HC, et al. Targeting triple-negative breast cancer cells with the histone deacetylase inhibitor panobinostat. Breast Cancer Res. 2012;14(3):R79.

74. Kai M, Kanaya N, Wu SV, et al. Targeting breast cancer stem cells in triple-negative breast cancer using a combination of LBH589 and salinomycin. Breast Cancer Res Treat. 2015;151(2):281-294.

75. Connolly RM, Jankowitz RC, Zahnow CA, et al. Abstract 4666 : A phase 2 study investigating the safety, efficacy and surrogate biomarkers of response of 5-azacitidine (5-AZA) andentinostat (MS275 ) in patients with triple-negative advanced breast cancer. Cancer Res 2013;73(supply 8):6-10.

76. Fuino L, Bali P, Wittmann S, et al. Histone deacetylase inhibitor LAQ824 down-regulates Her-2 and sensitizes human breast cancer cells to trastuzumab, taxotere, gemcitabine, and epothilone. Mol Cancer Ther. 2003;2(10):971-984.

77. Müller BM, Jana L, Kasajima A, et al. Differential expression of histone deacetylases HDAC1, 2 and 3 in human breast cancer - overexpression of HDAC2 and HDAC3 is associated with clinicopathological indicators of disease progression. BMC Cancer. 2013;13:215.

78. Kawai H, Li H, Avraham S, et al. Overexpression of histone deacetylase HDAC1 modulates breast cancer progression by negative regulation of estrogen receptor $\alpha$. Int J Cancer. 2003;107(3):353-358.

79. Hsieh CL, Ma HP, Su CM, et al. Alterations in histone deacetylase 8 lead to cell migration and poor prognosis in breast cancer. Life Sci. 2016;151:7-14.

80. Cao C, Vasilatos SN, Bhargava R, et al. Functional interaction of histone deacetylase 5 (HDAC5) and lysine-specific demethylase 1 (LSD1) promotes breast cancer progression. Oncogene. 2017;36(1):133-145.

81. Lapierre M, Linares A, Dalvai M, et al. Histone deacetylase 9 regulates breast cancer cell proliferation and the response to histone deacetylase inhibitors. Oncotarget. 2016;7(15):19693-19708.

82. Chatterjee N, Tenniswood M. The potential of histone deacetylase inhibitors in breast cancer therapy. Breast cancer management. 2015;4(2):85-97.

83. Singh TR, Shankar S, Srivastava RK. HDAC inhibitors enhance the apoptosis-inducing potential of TRAIL in breast carcinoma. Oncogene. 2005;24(29):4609-4623.

84. Evens AM, Balasubramanian S, Vose JM, et al. A phase I/II multicenter, open-label study of the oral histone deacetylase inhibitor abexinostat in relapsed/refractory lymphoma. Clin Cancer Res. 2016;22(5):10591066 